\begin{tabular}{|r|l|}
\hline \multicolumn{2}{|c|}{ Statistica Sinica Preprint No: SS-2020-0361 } \\
\hline Title & $\begin{array}{l}\text { Data Integration in High Dimension With Multiple } \\
\text { Quantiles }\end{array}$ \\
\hline Manuscript ID & SS-2020-0361 \\
\hline URL & http://www.stat.sinica.edu.tw/statistica/ \\
\hline DOI & $10.5705 /$ ss.202020.0361 \\
\hline Complete List of Authors & $\begin{array}{l}\text { Guorong Dai, } \\
\text { Ursula Müller and } \\
\\
\text { Raymond James Carroll }\end{array}$ \\
\hline Corresponding Author & Guorong Dai \\
\hline E-mail & rondai@stat.tamu.edu \\
\hline Notice: Accepted version subject to English editing. \\
\hline
\end{tabular}


Statistica Sinica

\title{
DATA INTEGRATION IN HIGH DIMENSION WITH MULTIPLE QUANTILES
}

\author{
Guorong Dai, Ursula U. Müller and Raymond J. Carroll \\ Department of Statistics, Texas A\&M University \\ College Station, TX 77843, USA
}

Abstract: This article deals with the analysis of high dimensional data that come from multiple sources ("experiments") and thus have different possibly correlated responses, but share the same set of predictors. The measurements of the predictors may be different across experiments. We introduce a new regression approach with multiple quantiles to select those predictors that affect any of the responses at any quantile level and to estimate the nonzero parameters. Our approach differs from established methods by being able to handle heterogeneity in data sets as well as heavy-tailed error distributions, two difficulties that are often encountered in complex data scenarios. Our estimator is a minimizer of a penalized objective function, which aggregates the data from the different experiments. We establish model selection consistency and asymptotic normality of the estimator. In addition we present an information criterion, which can also be used for consistent model selection. Simulations and two data applications illustrate the advantages of our method in recovering the underlying regression models, which comes from taking the group structure induced by the predictors across experiments and quantile levels into account. 
Key words and phrases: Data integration; High dimensional data; Information criterion; Penalized quantile regression.

\section{Introduction}

To set the stage for this work on data integration, consider $K$ data sets from $K$ different populations, where $K$ is some fixed number, with linear regression models

$$
Y_{k}=X_{k}^{\mathrm{T}} \alpha_{k}^{*}+U_{k} \quad(k=1, \ldots, K) .
$$

Here $Y_{k}$ is a scalar response, $X_{k}$ is a $p$-dimensional predictor, $\alpha_{k}^{*}$ is a $p$ dimensional parameter vector and $U_{k}$ is the error term. Zellner (1962) referred to this set of models as seemingly unrelated regressions and proposed the idea of estimating the regression parameters simultaneously using a generalized least squares method. The responses in model (1.1) are different, but dependent, while the predictors are the same in the $K$ data sets, but not their values. This is, for example, given if individuals are assessed through various responses from different experiments and the predictor values are measured in different ways (Gao and Carroll, 2017).

Model (1.1), with the assumption that $E\left(U_{k} \mid X_{k}\right)=0$, can also be written as a heterogenous linear regression model, i.e. as

$$
E\left(Y_{k}-X_{k}^{\mathrm{T}} \alpha_{k}^{*} \mid X_{k}\right)=0 \quad(k=1, \ldots, K)
$$


We consider the same scenario, but pursue a different approach. Instead of modeling the conditional mean of the response given the covariates, we assume heterogeneous linear regression models for the conditional quantiles $Q_{\tau_{m}}\left(X_{k}\right)$ at various quantile levels $\tau_{m}(m=1, \ldots, M)$, i.e.

$$
E\left\{I\left(Y_{k} \leq X_{k}^{\mathrm{T}} \theta_{k m}^{*}\right)-\tau_{m} \mid X_{k}\right\}=0 \quad(k=1, \ldots, K)
$$

where $I(\cdot)$ is the indicator function and $\theta_{k m}^{*}$ is a $p$-dimensional parameter vector. This is equivalent to

$$
\operatorname{pr}\left(Y_{k} \leq X_{k}^{\mathrm{T}} \theta_{k m}^{*} \mid X_{k}\right)=\operatorname{pr}\left\{Y_{k} \leq Q_{\tau_{m}}\left(X_{k}\right) \mid X_{k}\right\}=\tau_{m}
$$

$(m=1, \ldots, M ; k=1, \ldots, K)$. We are interested in the high dimensional data situation and therefore let the dimension $p=p_{n}$ of the parameter vector tend to infinity as the sample size $n$ increases. In addition, we assume that the data are sparse, i.e. most of the parameters are zero, which means that only a fraction of the predictors affect the responses.

An important goal is to identify the relevant predictors. One possible approach is to aggregate each predictor's effect in all experiments by forming groups. In our scenario all responses share the same set of predictors. Hence we have a natural group structure: the parameters of different quantiles and experiments that belong to the same predictor constitute a group; see Gao and Carroll (2017), who develop a group penalized estimation method using a pseudolikelihood. To handle the unspecified depen- 
dence between the responses in the $K$ experiments, they pool the marginal likelihoods and impose $L_{2}$-group penalization on the grouped parameters. The group penalty was introduced in a 1999 Australian National University Ph.D. thesis by S. Bakin and then applied to group selection questions by Yuan and Lin (2006). Gao and Carroll (2017) use it to select predictors that are influential in any of the experiments. The main tool in their article is the smoothly clipped absolute deviation penalty (Fan and Li, 2001). In addition, Gao and Carroll (2017) use the concept of the Bayesian information criterion to develop a pseudolikelihood information criterion that applies to the high dimensional scenario. The pseudolikelihood approach they employ is an important advance and useful when the distribution of the error can be modeled parametrically, which is not assumed in our case.

In this article we use a linear quantile regression approach based on model (1.2), i.e. we will not work with a likelihood, but with a different objective function. Quantile regression was introduced by Koenker and Bassett (1978); see also Koenker (2005). In contrast to classical regression, it provides a global picture of the predictors' effect on the distribution of the responses, while it is robust to heavy-tailed distributions. In high dimensional settings Belloni and Chernozhukov (2011) studied linear quantile regression with a Lasso penalty, Wang et al. (2012) proved selection consistency of linear quantile regression with nonconvex penalty functions, and 
Sherwood and Wang (2016) derived asymptotic properties of partially linear additive quantile regression with a nonconvex penalty. In addition to these articles on single quantile regression, Zou and Yuan (2008a) introduced a composite quantile regression approach, which considers multiple quantiles simultaneously. They assumed that the slopes are the same across quantiles and used the adaptive Lasso penalty from Zou (2006). Their method shares the oracle properties proposed in Fan and Li (2001). In another paper, which focuses on computation and not on theoretical properties, Zou and Yuan (2008b) propose a related approach for the heterogeneous scenario, i.e. when covariates and errors are dependent so that the slopes vary across quantiles. They consider multiple responses, but model just one single quantile for each response. Their method is able to detect non-zero slopes simultaneously. The two 2008 articles by Zou \& Yuan study settings with a fixed number of parameters. Recently the composite quantile approach of Zou and Yuan (2008a) was extended to high dimensional scenarios by Gu and Zou (2020). These authors assume that the slopes are the same across quantiles, i.e. homogeneity, so their approach does not apply to the heterogeneous models investigated by our research. Fan et al. (2016) studied quantile regression with multiple responses under a "transnormal" assumption, which requires that the responses and predictors can be transformed to a multivariate normal variable by marginal monotone functions. 
This is not required in our model. In Table 3 of Section 4 we consider a simulation setting with some binary predictors, which violates this assumption. While we are interested in identifying relevant predictors, Fan et al. (2016) focus on predicting responses and estimating correlation matrices.

Our goal is simultaneous variable selection with multiple quantiles across $K$ experiments. To take account of the unknown dependence structure between the responses in the different experiments, we integrate the data by summing up their quantile loss functions analogous to Gao and Carroll (2017), who pool the likelihood functions. Additionally, similar to Sherwood and Wang (2016), who conduct variable selection with multiple quantiles, we apply a nonconvex penalty on the $L_{1}$-norm of the coefficients related to each predictor, which represents the overall strength of the predictor across multiple experiments and quantiles. This penalty function takes the group structure into account and excludes covariates that have no impact on any of the responses at any of the quantile levels. Moreover, the $L_{1}$-norm is computationally convenient in quantile regression settings, thanks to Peng and Wang (2015), who provid a new "Quick Iterative Coordinate Descent" algorithm for solving nonconvex penalized quantile regression in high dimensions with no group structure. With modifications, their algorithm can be adapted to our approach; see Section 4.

Our work is largely motivated by the widespread existence of hetero- 
geneity in complex data sets (Yu et al., 2003; Wang et al., 2012; Lee et al., 2014) such as the liver toxicity data set and the financial index data analyzed in Section 5. In contrast to classical regression, which focuses on the conditional mean or on one single conditional quantile of $Y_{k}$ given $X_{k}$ $(k=1, \ldots, K)$, a major advantage of our approach is its ability to identify predictors in heterogeneous models that affect the responses at one or more quantile levels, but not necessarily globally. When the random errors in the data generating mechanism have a heavy-tailed distribution, e.g. a $t$-distribution with a small number of degrees of freedom, quantile based methods have a better estimation accuracy than competing approaches that use the quadratic loss function. Despite these clear advantages, multiple quantile regression for dependent data that originate from different sources has not, to the best of our knowledge, been studied in the literature. We also cover the high dimensional data scenario by adding a nonconvex group penalty term. We establish selection consistency and asymptotic normality of our estimator in this quite general setting under mild assumptions. Additionally we propose a multiple quantile Bayesian information criterion (MQBIC) based on pooled check functions, which is an extension of the Bayesian information criterion for linear quantile regression (Lee et al., 2014) to the multiple experiment scenario. Similar to the pseudolikelihood information criterion in Gao and Carroll (2017), MQBIC permits consistent 
model selection (see Section 3) and choice of the tuning parameter for the penalized estimator (see Section 4).

Summing up, the main contribution of this article is the introduction of quantile based methods to the high dimensional scenario of data integration. We propose a penalized estimation process and an information criterion, which can identify the covariates that affect any of the responses at any of the quantile levels. Our method enjoys robustness and can be applied to the complex scenario with heterogeneous data and dependent responses.

The rest of this article is organized as follows. In Section 2 we introduce our objective function, which involves a nonconvex group penalization term, and present the oracle properties of the estimator. The MQBIC is presented in Section 3 and its model selection consistency is established. In Section 4 we compare our method with other approaches using simulations. Our method is illustrated in Section 5 by means of empirical data examples. Section 6 gives a brief discussion of further questions. All proofs as well as additional simulation results are in the Supplementary Material. For notational clarity we assume in the following that the sample sizes and the quantile levels are the same in every experiment. The conclusions and methods are essentially the same if we drop these assumptions. 


\section{Penalized estimator}

Throughout this article we will use the capital letter $C$ to represent a generic constant, including $C_{1}, C_{2}$, etc. We write $I_{m}$ for the $m \times m$ identity matrix. The symbols $\|\cdot\|_{1}$ and $\|\cdot\|$ refer to the $L_{1}$ and $L_{2}$-norms of a vector and $\otimes$ denotes the Kronecker product.

Our conditional quantile regression model is $Q_{\tau_{m}}\left(X_{k}\right)=X_{k}^{\mathrm{T}} \theta_{k m}^{*}$ with ordered levels $0<\tau_{1}<\tau_{2}<\cdots<\tau_{M}<1$. We can set the first column of $X_{k}$ to be $(1, \ldots, 1)^{\mathrm{T}}$ so that the model contains intercept terms. For notational convenience, we assume the intercepts all equal zero. The number of predictors $p=p_{n}$ tends to infinity as the sample size $n$ increases.

For $k=1, \ldots, K$ and $i=1, \ldots, n$ we consider $n$ independent copies $\left\{Y_{k i}, X_{k i}\right\}$ with $X_{k i}=\left(X_{k i 1}, \ldots, X_{k i p_{n}}\right)^{\mathrm{T}}$ of the base observation $\left\{Y_{k}, X_{k}\right\}$ from model (1.1). Here we use three subscripts to locate the predictors, i.e. $X_{k i j}$ represents the $j^{\text {th }}$ component of the $i^{\text {th }}$ observation in the $k^{\text {th }}$ experiment. We write $X_{k \cdot j}=\left(X_{k 1 j}, \ldots, X_{k n j}\right)^{\mathrm{T}}$ for the vector. The data are summarized in Table 1.

The regression parameters $\theta_{k m}^{*}(k=1, \ldots, K, m=1, \ldots, M)$ are assumed to be sparse, i.e. most of the components of $\theta_{k m}^{*}$ are zero. Write $\theta^{*(j)}$ for the parameters related to the $j^{\text {th }}$ predictor $\left(j=1, \ldots, p_{n}\right)$ across the $K$ 
experiments and the $M$ quantile levels, i.e.

$$
\theta^{*(j)}=\left(\theta_{11 j}^{*}, \ldots, \theta_{1 M j}^{*}, \ldots, \theta_{K 1 j}^{*}, \ldots, \theta_{K M j}^{*}\right)^{\mathrm{T}}
$$

We want to select the predictors that have an effect on any of the responses, i.e. we want to specify the set $\mathcal{A}=\left\{j: 1 \leq j \leq p_{n},\left\|\theta^{*(j)}\right\|>0\right\}$. Without loss of generality let $\mathcal{A}=\left\{1,2, \ldots, q_{n}\right\}$, i.e. only the first $q_{n}$ predictors have nonzero parameters. We assume that $q_{n}$ tends to infinity as $n$ and $p_{n}$ increase. For convenience of notation, we use the letter $a$ at the end of a subscript if we refer to subvectors or submatrices that consist of components with subscripts in $\mathcal{A}$. For example, $X_{k i a}=\left(X_{k i 1}, \ldots, X_{k i q_{n}}\right)^{\mathrm{T}}, X_{k \cdot a}=$ $\left(X_{k 1 a}, \ldots, X_{k n a}\right)^{\mathrm{T}}$ and $\theta_{k m a}^{*}=\left(\theta_{k m 1}^{*}, \ldots, \theta_{k m q_{n}}^{*}\right)^{\mathrm{T}}$.

The dependence between the experiments is unspecified. To integrate the data we therefore sum up the quantile loss functions across the $K$ experiments and the $M$ quantiles,

$$
\ell_{n}(\theta)=n^{-1} \sum_{k=1}^{K} \sum_{m=1}^{M} \sum_{i=1}^{n} \rho_{m}\left(Y_{k i}-X_{k i}^{\mathrm{T}} \theta_{k m}\right) .
$$

In the above $\rho_{m}(x)=x\left\{\tau_{m}-I(x<0)\right\}$ is the check function and

$$
\theta=\left(\theta_{11}^{\mathrm{T}}, \ldots, \theta_{1 M}^{\mathrm{T}}, \ldots, \theta_{K 1}^{\mathrm{T}}, \ldots, \theta_{K M}^{\mathrm{T}}\right)^{\mathrm{T}}
$$

is a parameter vector. Loss functions analogous to (2.3) with $K=1$ or $M=1$ were used for low dimensional linear models by Zou and Yuan (2008b). The big difference between (2.3) and the composite quantile loss 
Table 1: Data structure of multiple experiments

\begin{tabular}{|c|c|c|c|}
\hline & Experiment 1 & $\ldots$ & Experiment $K$ \\
\hline$\tau_{1}$ & $\theta_{11}^{*}=\left(\theta_{111}^{*}, \ldots, \theta_{11 p_{n}}^{*}\right)^{\mathrm{T}}$ & $\cdots$ & $\theta_{K 1}^{*}=\left(\theta_{K 11}^{*}, \ldots, \theta_{K 1 p_{n}}^{*}\right)^{\mathrm{T}}$ \\
\hline$\tau_{M}$ & $\theta_{1 M}^{*}=\left(\theta_{1 M 1}^{*}, \ldots, \theta_{1 M p_{n}}^{*}\right)^{\mathrm{T}}$ & $\cdots$ & $\theta_{K M}^{*}=\left(\theta_{K M 1}^{*}, \ldots, \theta_{K M p_{n}}^{*}\right)^{\mathrm{T}}$ \\
\hline$i=1$ & $Y_{11}, X_{11}=\left(X_{111}, \ldots, X_{11 p_{n}}\right)^{\mathrm{T}}$ & $\cdots$ & $Y_{K 1}, X_{K 1}=\left(X_{K 11}, \ldots, X_{K 1 p_{n}}\right)^{\mathrm{T}}$ \\
\hline$i=n$ & $Y_{1 n}, X_{1 n}=\left(X_{1 n 1}, \ldots, X_{1 n p_{n}}\right)^{\mathrm{T}}$ & $\ldots$ & $Y_{K n}, X_{K n}=\left(X_{K n 1}, \ldots, X_{K n p_{n}}\right)^{\mathrm{T}}$ \\
\hline
\end{tabular}

Parameters related to $\tau_{1}, \ldots, \tau_{M}$ and observations $i=1$ to $n$.

function considered in Zou and Yuan (2008a) and Gu and Zou (2020) is that we allow $\theta_{k m} \neq \theta_{k m^{\prime}}\left(1 \leq m \neq m^{\prime} \leq M ; k=1, \ldots, K\right)$, i.e. that the slopes are different.

To select the predictors that affect any of the responses, a nonconvex penalty function $\Omega_{\lambda_{n}}(\cdot)$ with tuning parameter $\lambda_{n}$ is imposed on the overall impact of each predictor. That impact is represented by the $L_{1}$ norm of the vector $\theta^{(j)}$, which contains the parameters of the $j^{\text {th }}$ predictor in the $K$ experiments. This gives the overall objective function

$$
\Gamma_{\lambda_{n}}(\theta)=\ell_{n}(\theta)+\sum_{j=1}^{p_{n}} \Omega_{\lambda_{n}}\left(\left\|\theta^{(j)}\right\|_{1}\right)
$$

Our estimator is obtained by minimizing $\Gamma_{\lambda_{n}}(\theta)$. We use the smoothly 
clipped absolute deviation (SCAD) penalty function (Fan and Li, 2001)

$$
\begin{aligned}
\Omega_{\lambda_{n}}(x)= & \lambda_{n} x I\left(0 \leq x \leq \lambda_{n}\right)+ \\
& \frac{a \lambda_{n} x-\left(x^{2}+\lambda_{n}^{2}\right) / 2}{a-1} I\left(\lambda_{n}<x<a \lambda_{n}\right)+\frac{(a+1) \lambda_{n}^{2}}{2} I\left(x \geq a \lambda_{n}\right),
\end{aligned}
$$

where $a$ is a constant that is usually set to 3.7 (Fan and Li, 2001). Before stating the asymptotic properties of our estimator, we make the following assumptions.

Assumption 1. There is a constant $C>0$ such that $\left|X_{k i j}\right| \leq C$ for every $k=1, \ldots, K, i=1, \ldots, n$ and $j=1, \ldots, p_{n}$.

Assumption 2. For every $k=1, \ldots, K$ there are positive constants $C_{1}$ and $C_{2}$ such that

$$
C_{1} \leq \lambda_{\min }\left(n^{-1} X_{k \cdot a}^{\mathrm{T}} X_{k \cdot a}\right) \leq \lambda_{\max }\left(n^{-1} X_{k \cdot a}^{\mathrm{T}} X_{k \cdot a}\right) \leq C_{2}
$$

where $\lambda_{\min }(\cdot)$ and $\lambda_{\max }(\cdot)$ stand for the smallest and the largest eigenvalue, respectively. In addition $X_{k \cdot a}$ and $\left(Y_{k 1}, \ldots, Y_{k n}\right)^{\mathrm{T}}$ are in "general positions", which is an identifiability condition that guarantees that a solution to the quantile regression problem exists (Koenker, 2005, Section 2.2.2).

Assumption 3. For every $k=1, \ldots, K$ and $m=1, \ldots, M$, the conditional probability density $f_{k m}(\cdot \mid x)$ of $\varepsilon_{k m}=Y_{k}-X_{k}^{\mathrm{T}} \theta_{k m}^{*}$ given $X_{k}=x$ is uniformly bounded and bounded away from zero in a neighborhood of zero, and has a derivative $f_{k m}^{\prime}(\cdot \mid x)$, which is uniformly bounded in a neighborhood of zero. 
Assumption 4. The true model size satisfies $q_{n}=O\left(n^{c_{1}}\right)$ for some $0 \leq$ $c_{1}<1 / 2$.

Assumption 5. There are positive constants $c_{2}$ and $C$ such that $2 c_{1}<$ $c_{2} \leq 1$, where $c_{1}$ is the constant introduced in Assumption 4, and

$$
n^{\left(1-c_{2}\right) / 2} \min _{1 \leq j \leq q_{n}}\left\|\theta^{*(j)}\right\|_{1} \geq C .
$$

Assumptions 1 and 2 guarantee good behavior of the design matrices. Assumption 1 could be relaxed by letting the covariate values increase to infinity at a certain slow rate. We work with it mainly for reasons of simplicity and clarity; see e.g. the closely related articles by Wang et al. (2012), Lee et al. (2014) and Sherwood and Wang (2016), who also limit themselves to the case with bounded covariates. The conditions in Assumption 3 concern the unknown distribution of the random errors. They are considerably weaker than assuming a specific parametric model for the error distribution. Assumption 4 regulates the growth rate of the true model size. This is a standard assumption to establish asymptotic properties of sparse estimators in linear models with a diverging number of parameters; see, for example, Wang et al. (2012) and Lee et al. (2014). In addition, it is weaker than the condition $q_{n}=o\left(n^{1 / 5}\right)$ required by Gao and Carroll (2017). Assumption 5 excludes situations where the nonzero parameters decay too fast. Conditions similar to Assumptions 1-5 were required in Wang et al. 
(2012) for single experiments with a single quantile.

The oracle estimator $\hat{\theta}$ is defined as the minimizer of $\ell_{n}(\theta)$ that knows that the first $q_{n}$ components of $\theta$ are nonzero and that the others are zero, i.e. $\left\|\widehat{\theta}^{(j)}\right\|=0$ for $q_{n}<j \leq p_{n}$. The following theorem provides the model selection consistency of our estimator. More precisely, we will show that, with probability tending to one, the oracle estimator can be obtained with our approach, i.e. by minimizing the objective function $\Gamma_{\lambda_{n}}(\theta)$.

Theorem 1. Let $S\left(\lambda_{n}\right)$ denote the set of local minimizers of $\Gamma_{\lambda_{n}}(\theta)$ and $\widehat{\theta}$ the oracle estimator. Under Assumptions 1-5, $\operatorname{pr}\left\{\widehat{\theta} \in S\left(\lambda_{n}\right)\right\} \rightarrow 1$ as $n \rightarrow \infty$, if $\lambda_{n}=o\left\{n^{-\left(1-c_{2}\right) / 2}\right\}, n^{-1 / 2} q_{n}=o\left(\lambda_{n}\right)$ and $n^{-1} \log p_{n}=o\left(\lambda_{n}^{2}\right)$.

Before stating Theorem 2, we introduce some notation. We write

$$
\begin{aligned}
& \varepsilon_{k m i}=Y_{k i}-X_{k i}^{\mathrm{T}} \theta_{k m}^{*}, \quad \varepsilon_{k m}=\left(\varepsilon_{k m 1}, \ldots, \varepsilon_{k m n}\right)^{\mathrm{T}}, \\
& \varepsilon=\left(\varepsilon_{11}^{\mathrm{T}}, \ldots, \varepsilon_{1 M}^{\mathrm{T}}, \ldots, \varepsilon_{K 1}^{\mathrm{T}}, \ldots, \varepsilon_{K M}^{\mathrm{T}}\right)^{\mathrm{T}}, \\
& \psi_{k m i}(\varepsilon)=\tau_{m}-I\left(\varepsilon_{k m i}<0\right), \quad \psi_{n k m}(\varepsilon)=\left\{\psi_{k m 1}(\varepsilon), \ldots, \psi_{k m n}(\varepsilon)\right\}^{\mathrm{T}}, \\
& \psi_{n k}(\varepsilon)=\left\{\psi_{n k 1}(\varepsilon)^{\mathrm{T}}, \ldots, \psi_{n k M}(\varepsilon)^{\mathrm{T}}\right\}^{\mathrm{T}}, \quad \psi_{n}(\varepsilon)=\left\{\psi_{n 1}(\varepsilon)^{\mathrm{T}}, \ldots, \psi_{n K}(\varepsilon)^{\mathrm{T}}\right\}^{\mathrm{T}}, \\
& H_{n}=E\left\{\psi_{n}(\varepsilon) \psi_{n}(\varepsilon)^{\mathrm{T}} \mid \mathcal{X}\right\} \text { with } \mathcal{X}=\left\{X_{k i}: k=1, \ldots, K, i=1, \ldots, n\right\},
\end{aligned}
$$


where $k=1, \ldots, K, m=1 \ldots, M$ and $i=1, \ldots, n$. Further we set

$$
\begin{aligned}
& B_{n k m}=\operatorname{diag}\left\{f_{k m}\left(0 \mid X_{k 1}\right), \ldots, f_{k m}\left(0 \mid X_{k n}\right)\right\} \\
& B_{n}=\operatorname{diag}\left(B_{n 1}, \ldots, B_{n K}\right) \text { with } B_{n k}=\operatorname{diag}\left(B_{n k 1}, \ldots, B_{n k M}\right), \\
& \theta_{a}^{*}=\left(\theta_{11 a}^{* \mathrm{~T}}, \ldots, \theta_{1 M a}^{* \mathrm{~T}}, \ldots, \theta_{K 1 a}^{* \mathrm{~T}}, \ldots, \theta_{K M a}^{* \mathrm{~T}}\right)^{\mathrm{T}}, \\
& \widehat{\theta}_{k m a}=\left(\widehat{\theta}_{k m 1}, \ldots, \widehat{\theta}_{k m q_{n}}\right)^{\mathrm{T}}, \widehat{\theta}_{a}=\left(\widehat{\theta}_{11 a}^{\mathrm{T}}, \ldots, \widehat{\theta}_{1 M a}^{\mathrm{T}}, \ldots, \widehat{\theta}_{K 1 a}^{\mathrm{T}}, \ldots, \widehat{\theta}_{K M a}^{\mathrm{T}}\right)^{\mathrm{T}}, \\
& X_{a}=\operatorname{diag}\left(I_{M} \otimes X_{1 \cdot a}, \ldots, I_{M} \otimes X_{K \cdot a}\right), R_{n}=n^{-1} X_{a}^{\mathrm{T}} B_{n} X_{a}, \\
& S_{n}=n^{-1} X_{a}^{\mathrm{T}} H_{n} X_{a}, \Sigma_{n}=R_{n}^{-1} S_{n} R_{n}^{-1} .
\end{aligned}
$$

The next theorem gives the asymptotic normality of low dimensional projections of the nonzero part $\widehat{\theta}_{a}$ of the oracle estimator $\widehat{\theta}$ from Theorem 1 . An illustration of the result with histogram plots (for two simulation scenarios from Section 4) is provided in Section S3 of the Supplementary Material.

Theorem 2. Let $q_{n}^{*}=q_{n} \times M \times K$. Consider a $s \times q_{n}^{*}$ matrix $A_{n}$ with $s$ fixed and $A_{n} A_{n}^{\mathrm{T}} \rightarrow G$, a positive definite matrix, then

$$
n^{1 / 2} A_{n} \Sigma_{n}^{-1 / 2}\left(\widehat{\theta}_{a}-\theta_{a}^{*}\right) \rightarrow N(\mathbf{0}, G) \quad(n \rightarrow \infty)
$$

in distribution, provided Assumptions 1-4 are satisfied and $\lambda_{\min }\left(S_{n}\right)$ is uniformly bounded away from zero.

Theorems 1 and 2 establish the model selection consistency and asymptotic normality of our estimator when experiments are correlated. This 
shows that it is reasonable to aggregate information from multiple experiments, rather than ignoring the correlation and analyzing each experiment separately.

\section{Multiple quantile Bayesian information criterion}

To select the correct model we use an information criterion that balances the goodness-of-fit and the complexity of a model. By applying this information criterion to a set of competing models, the true model can be identified with probability approaching one. In the context of quantile regression, Lee et al. (2014) develop a Bayesian information criterion with a diverging number of predictors. That method considers one single quantile and deals with data from one single experiment. We use a generalized version of the criterion, now based on multiple quantiles and on data from several experiments, which improves its ability to select the correct model.

The multiple quantile Bayesian information criterion of a submodel $\mathcal{D} \subset$ $\left\{1,2, \ldots, p_{n}\right\}$ is

$$
\begin{aligned}
\operatorname{MQBIC}(\mathcal{D})= & \log \left\{\sum_{k=1}^{K} \sum_{m=1}^{M} \sum_{i=1}^{n} \rho_{m}\left(Y_{k i}-X_{k i \mathcal{D}}^{\mathrm{T}} \widehat{\theta}_{k m \mathcal{D}}\right)\right\} \\
& +(2 n)^{-1}|\mathcal{D}| T_{n} \log n
\end{aligned}
$$

where $\widehat{\theta}_{k m \mathcal{D}}=\arg \min _{\theta \in \mathbb{R}^{|\mathcal{D}|}} \sum_{i=1}^{n} \rho_{m}\left(Y_{k i}-X_{k i \mathcal{D}}^{\mathrm{T}} \theta\right)$ for $k=1, \ldots, K$ and $m=1, \ldots, M,|\mathcal{D}|$ is the cardinality of $\mathcal{D}$, and $T_{n}$ is a sequence of positive 
constants diverging to infinity as $n$ increases. The notation $X_{k i \mathcal{D}}$ refers to the subvectors of $X_{k i}$. which only contain the components with subscripts in $\mathcal{D}$. We set an upper bound on the cardinality of competing models, say $d_{n}$, and search for the best model among submodels whose cardinality is smaller or equal to $d_{n}$. Define $\mathcal{D}^{*}=\left\{1,2, \ldots, q_{n}\right\}$ as the subset of $\left\{1, \ldots, p_{n}\right\}$ corresponding to the true model, and $\mathcal{M}=\left\{\mathcal{D} \subset\left\{1, \ldots, p_{n}\right\}:|\mathcal{D}| \leq\right.$ $\left.d_{n}\right\}$ as the set of all competing models. The first part of the MQBIC represents the goodness-of-fit, while the second term is a penalty on the model complexity. To guarantee model selection consistency of the MQBIC we need the following assumptions, in addition to some of the assumptions from Section 2 .

Assumption 6. For every $k=1, \ldots, K$ there are constants $0<C_{3} \leq C_{4}$ such that for any $\mathcal{D} \subset\left\{1, \ldots, p_{n}\right\}$ the matrix $X_{k \cdot \mathcal{D}}=\left(X_{k 1 \mathcal{D}}, \ldots, X_{k n \mathcal{D}}\right)^{\mathrm{T}}$ satisfies

$C_{3} \leq \min _{|\mathcal{D}| \leq 2 d_{n}} \lambda_{\min }\left(n^{-1} X_{k \cdot \mathcal{D}}^{\mathrm{T}} X_{k \cdot \mathcal{D}}\right) \leq \max _{|\mathcal{D}| \leq 2 d_{n}} \lambda_{\max }\left(n^{-1} X_{k \cdot \mathcal{D}}^{\mathrm{T}} X_{k \cdot \mathcal{D}}\right) \leq C_{4}$

Assumption 7. The full model size $p_{n}$ is of order $p_{n}=O\left(n^{c_{3}}\right)$ for some $c_{3}>0$; the true model size $q_{n}$ is fixed, $q_{n}=q$, and satisfies $q \leq d_{n}=O\left(n^{c_{4}}\right)$ for some $0<c_{4}<1 / 2$.

Assumption 8. The sequence $T_{n}$ in the definition (3.5) satisfies $T_{n} \rightarrow \infty$ and $n^{-1} T_{n} \log n \rightarrow 0$. 
Assumption 9. The average $n^{-1} \sum_{k=1}^{K} \sum_{m=1}^{M} \sum_{i=1}^{n} \rho_{m}\left(\varepsilon_{k m i}\right)$ of the check functions is bounded away from zero with probability tending to one.

Assumption 6 extends Assumption 2 for the true model to all candidate models. This is common for scenarios with more regression parameters than observations, i.e. $p_{n}>n$. In Assumption 7, the true model size is fixed because of a technical difficulty in handling the maximum of $\left|\mathcal{D} \backslash \mathcal{D}^{*}\right|^{-1}\left|n^{-1} \sum_{i=1}^{n}\left\{\rho_{m}\left(Y_{k i}-X_{k i \mathcal{D}}^{\mathrm{T}} \widehat{\theta}_{k m \mathcal{D}}\right)-\rho_{m}\left(Y_{k i}-X_{k i \mathcal{D}^{*}}^{\mathrm{T}} \widehat{\theta}_{k m \mathcal{D}^{*}}\right)\right\}\right|$ over the set of overfitted models $\left\{\mathcal{D} \in \mathcal{M}: \mathcal{D}^{*} \subset \mathcal{D}, \mathcal{D} \neq \mathcal{D}^{*}\right\}$ (Lee et al., 2014). Assumption 8 regulates the growth rate of the sequence $T_{n}$. Assumption 9 is made for convenience in the proofs because $n^{-1} \sum_{k=1}^{K} \sum_{m=1}^{M} \sum_{i=1}^{n} \rho_{m}\left(\varepsilon_{k m i}\right)$ appears in denominators.

In the following theorem we show that the true model has, with probability tending to one, the smallest MQBIC value among all candidate models.

Theorem 3. If Assumptions 1, 3 and 6-9 hold, then with probability tending to one, the true model can be selected by minimizing the $M Q B I C$, that is

$$
\lim _{n \rightarrow \infty} \operatorname{pr}\left\{\min _{\mathcal{D} \in\left(\mathcal{M} \backslash\left\{\mathcal{D}^{*}\right\}\right)} M Q B I C(\mathcal{D})>M Q B I C\left(\mathcal{D}^{*}\right)\right\}=1
$$

Theorem 3 establishes model selection consistency of the MQBIC for data from multiple dependent sources, which provides another approach to identify the true underlying model. In the MQBIC approach estimation and 
model selection are separate processes. This is different from minimizing the objective function in Section 2, which is a one-step procedure. The main advantage of the MQBIC is that we can use it to select the tuning parameter $\lambda_{n}$ for the penalized estimation process in Section 2, which is computationally more efficient than cross validation. The details are given in Section 4.

\section{Simulations}

In this section we study the numerical performance of our estimators. We use the objective function (2.4) with $M=5$ quantiles, $\tau_{1}=1 / 6, \tau_{2}=$ $2 / 6, \ldots, \tau_{5}=5 / 6$, and study two different group structures, namely complete and incomplete grouping. Complete grouping means that parameters of the same predictor can only be either all zero or all nonzero, while in the incomplete case a group may contain both zero and nonzero parameters.

In both cases the number of experiments is $K=2$, while the sample size and the number of predictors are $(n, p)=(100,100),(100,200)$ or $(200,1000)$. The nonzero parameters are drawn independently from a uniform distribution on $[0.05,1]$. For $K=1,2$ we generate independent random vectors $X_{k i}^{\prime}, i=1, \ldots, n$, from a $p$-dimensional multivariate normal distribution with mean zero and a covariance matrix whose $(i, j)^{\text {th }}$ component is $0.5^{|i-j|}$ for $1 \leq i, j \leq p$. The predictors $X_{k i}$ for the different 
scenarios described below are transformations of the $X_{k i}^{\prime}$ 's. For $i=1, \ldots, n$ the error terms $\left(\xi_{1 i}, \xi_{2 i}\right)^{\mathrm{T}}$ are drawn independently from a bivariate normal distribution with mean zero or from a bivariate $t$-distribution with three degrees of freedom. The covariance matrix of $\left(\xi_{1 i}, \xi_{2 i}\right)$ is $\Sigma$ with entries $\Sigma_{11}=\Sigma_{22}=1$ and $\Sigma_{12}=\Sigma_{21}=0.7$

For minimizing the objective function (2.4) with a fixed $\lambda_{n}=\lambda$, we use an algorithm developed by Peng and Wang (2015) for penalized quantile regression, modified for our scenario with multiple quantiles and experiments. We first apply the "Majorize-Minimization" algorithm with an initial value $\widehat{\theta}(0)=\mathbf{0}$. Let $\widehat{\theta}(r-1)$ denote the result from the $(r-1)$ th iteration. According to Section 3.1 of Peng and Wang (2015), the objective function (2.4) is majorized by

$$
\ell_{n}(\theta)+\sum_{j=1}^{p} \Omega_{\lambda}^{\prime}\left(\left\|\widehat{\theta}^{(j)}(r-1)\right\|_{1}+\right)\left\|\theta^{(j)}\right\|_{1}=\sum_{k=1}^{K} \sum_{m=1}^{M} Q_{\widehat{\theta}(r-1)}^{(k, m)}\left(\theta_{k m}\right)
$$

at the $r$ th iteration. Here $\Omega_{\lambda}^{\prime}(\cdot)$ is the derivative of $\Omega_{\lambda}(\cdot)$ with $\Omega_{\lambda}^{\prime}\left(x_{0}+\right)=$ $\lim _{x \downarrow x_{0}} \Omega_{\lambda}^{\prime}(x)$ and

$$
\begin{aligned}
Q_{\widehat{\theta}(r-1)}^{(k, m)}\left(\theta_{k m}\right)= & n^{-1} \sum_{i=1}^{n} \rho_{m}\left(Y_{k i}-X_{k i}^{\mathrm{T}} \theta_{k m}\right)+ \\
& \sum_{j=1}^{p} \Omega_{\lambda}^{\prime}\left(\left\|\widehat{\theta}^{(j)}(r-1)\right\|_{1}+\right)\left|\theta_{k m j}\right|
\end{aligned}
$$

The minimization of the majorizing function (4.6) can therefore be done by minimizing $Q_{\widehat{\theta}(r-1)}^{(k, m)}\left(\theta_{k m}\right)(k=1, \ldots, K ; m=1, \ldots, M)$ separately for 
each $(k, m)$, using the "coordinate descent algorithm", which involves the calculation of weighted medians by the "quicksort" algorithm. A detailed description can be found in Section 3.2 of Peng and Wang (2015). We update $\widehat{\theta}(r-1)$ by

$$
\widehat{\theta}(r)=\arg \min _{\theta} \sum_{k=1}^{K} \sum_{m=1}^{M} Q_{\widehat{\theta}(r-1)}^{(k, m)}\left(\theta_{k m}\right)
$$

and repeat this process until convergence. This yields the minimizer $\widehat{\theta}_{\lambda, k m}=$ $\left(\widehat{\theta}_{\lambda, k m 1}, \ldots, \widehat{\theta}_{\lambda, k m p}\right)^{\mathrm{T}}(k=1, \ldots, K ; m=1, \ldots, M)$ of $(2.4)$ with $\lambda_{n}=\lambda$. The tuning parameter is chosen from a grid $\Lambda$. For $\lambda \in \Lambda$ let $\mathcal{D}_{\lambda}=\{j: 1 \leq$ $\left.j \leq p, \sum_{k=1}^{K} \sum_{m=1}^{M}\left|\widehat{\theta}_{\lambda, k m j}\right|>0\right\}$. For the final estimator we use

$$
\begin{aligned}
\widehat{\lambda}=\arg \min _{\lambda \in \Lambda}[ & \log \left\{\sum_{k=1}^{K} \sum_{m=1}^{M} \sum_{i=1}^{n} \rho_{m}\left(Y_{k i}-X_{k i}^{\mathrm{T}} \widehat{\theta}_{\lambda, k m}\right)\right\} \\
& \left.+(2 n)^{-1}\left|\mathcal{D}_{\lambda}\right|(\log n) T\right]
\end{aligned}
$$

which minimizes the MQBIC. This approach adapts criterion (2.10) in Lee et al. (2014) to multiple quantile levels and experiments. Since that article recommends $T=C \log p$ and their simulation results show that this type of information criterions tends to underfit models slightly, we consider $T=$ $(\log p) / 3$ or $(\log p) / 6$ and examine how this affects the performance of the method.

In each scenario we record the following three indices.

1. Positive selection rate (PSR): the proportion of selected predictors that affect any quantile of any response. Then, formally, PSR = 
$|\widehat{\mathcal{A}} \cap \mathcal{A}| /|\mathcal{A}|$ with $\mathcal{A}=\left\{j: 1 \leq j \leq p,\left\|\theta^{*(j)}\right\|>0\right\}$ and $\widehat{\mathcal{A}}=\{j: 1 \leq$ $\left.j \leq p,\left\|\widehat{\theta}^{(j)}\right\|>0\right\}$.

2. False discovery rate (FDR): the proportion of selected predictors that affect no response, i.e. $\left|\widehat{\mathcal{A}} \cap \mathcal{A}^{c}\right| /\left|\mathcal{A}^{c}\right|$.

3. Absolute error (AE): the absolute estimation error $(K M)^{-1}\left\|\widehat{\theta}-\theta^{*}\right\|_{1}$.

Our data integration (DI) approach is compared with the following three methods.

(a) Combined analysis based on the $\tau^{\text {th }}$ quantile $(\mathrm{CA}-\tau)$. This method considers only one quantile $\tau$. The data from the $K$ experiments are analyzed separately, then the $K$ sets of selected predictors are merged. We will see that in most of the cases the CA- $\tau$ method selects more unimportant predictors than our DI approach. Hence the false discovery rates will rise even further when the results from different quantile levels are combined.

(b) Sparse canonical correlation analysis (SCCA) by Witten and Tibshirani (2009). To adapt this method for data integration problems, we determine sparse vectors

$$
\left\{\widehat{w}_{1}, \widehat{w}_{2}\right\}=\arg \max _{\left\{w_{1}, w_{2}\right\}} \sum_{i=1}^{n} w_{1}^{\mathrm{T}} \tilde{X}_{i} \tilde{Y}_{i}^{\mathrm{T}} w_{2}
$$


that satisfy

$$
\left\|w_{1}\right\| \leq 1,\left\|w_{2}\right\| \leq 1,\left\|w_{1}\right\|_{1} \leq c_{1},\left\|w_{2}\right\| \leq c_{2}
$$

where $c_{1}$ and $c_{2}$ are some approriate tuning parameters and

$\tilde{X}_{i}=\left(X_{1 i}^{\mathrm{T}}, X_{2 i}^{\mathrm{T}}, \ldots, X_{K i}^{\mathrm{T}}\right)^{\mathrm{T}}, \tilde{Y}_{i}=\left(Y_{1 i}, Y_{2 i}, \ldots, Y_{K i}\right)^{\mathrm{T}} \quad(i=1, \ldots, n)$.

Then we view the predictors corresponding to the nonzero components of $\widehat{w}_{1}$ as the selected ones. Details of the implementation can be found in Witten and Tibshirani (2009). This approach does not generate estimators for the regression parameters, which explains why we have no values for the absolute errors (AE) in the tables below.

(c) The data integration method based on the least squares regression and the SCAD penalty (DI-LS) proposed by Gao and Carroll (2017), which we explained in the introduction. This method minimizes an objective function similar to (2.4), but with $M=1$ and $\ell_{n}(\cdot)$ replaced by the quadratic loss function, while the SCAD penalty is applied to $\left\|\theta^{(j)}\right\|$ $\left(j=1, \ldots, p_{n}\right)$. When calculating absolute errors, the target parameter is the vector $\tilde{\theta}_{k}$ that satisfies $E\left(Y_{k} \mid X_{k}\right)=X_{k}^{\mathrm{T}} \widetilde{\theta}_{k}(k=1, \ldots, K)$.

The value of $T$ only plays a role for minimizing the MQBIC. Hence it only affects our DI and the CA- $\tau$ methods in the following tables. The tuning parameters in SCCA and DI-LS are selected by 10-fold cross validation. 
Table 2: Positive selection rates, false discovery rates and absolute errors of the data integration method and the combined analysis for models with normal errors and complete group structure. Here DI denotes the data integration method based on multiple quantiles, CA- $\tau$ the combined analysis with one quantile $\tau=2 / 6$ or $3 / 6$, SCCA the sparse canonical correlation analysis, DI-LS the data integration method based on the least squares regression; PSR is the positive selection rate, FDR the false discovery rate and $\mathrm{AE}$ the absolute error $(K M)^{-1}\left\|\widehat{\theta}-\theta^{*}\right\|_{1}$. The parameter $T$ in criterion (4.7) equals $(\log p) / 3$ or $(\log p) / 6$. The sample and model sizes are $(\mathrm{a})(n, p)=(100,100)$, (b) $(100,200)$ or (c) $(200,1000)$. models with the incomplete group structure. Here $p$, the full model size; DI, data integration method; CA- $\tau$, combined analysis with $\tau$ quantile; PSR, positive selection rate; FDR, false discovery rate; AE, absolute error $(K M)^{-1} \sum_{k=1}^{K} \sum_{m=1}^{M} \sum_{j=1}^{p}\left|\widehat{\theta}_{k m j}-\theta_{k m j}^{*}\right|$. The parameter $T$ in the criterion (4.7) is (a) $\log p$ or (b) $(\log p) / 6$.

\begin{tabular}{|c|c|c|c|c|c|c|}
\hline \multirow[b]{2}{*}{ (a) } & \multicolumn{3}{|c|}{$T=(\log p) / 3$} & \multicolumn{3}{|c|}{$T=(\log p) / 6$} \\
\hline & $\operatorname{PSR}(\%)$ & $\operatorname{FDR}(\%)$ & $\mathrm{AE}$ & $\operatorname{PSR}(\%)$ & $\operatorname{FDR}(\%)$ & $\mathrm{AE}$ \\
\hline DI & $98.3(5.0)$ & $1.1(1.5)$ & $0.3(0.1)$ & $99.0(4.0)$ & $1.9(2.4)$ & $0.2(0.1)$ \\
\hline $\mathrm{CA}-(2 / 6)$ & $83.3(7.5)$ & $2.4(2.2)$ & $0.6(0.1)$ & $92.3(8.7)$ & $19.2(16.2)$ & $0.8(0.3)$ \\
\hline $\mathrm{CA}-(3 / 6)$ & $81.7(5.0)$ & $1.4(1.4)$ & $0.3(0.1)$ & $83.3(4.1)$ & $6.9(8.7)$ & $0.3(0.2)$ \\
\hline SCCA & $53.5(15.0)$ & $4.4(3.7)$ & & & & \\
\hline DI-LS & $85.0(6.5)$ & $5.9(5.0)$ & $0.3(0.1)$ & & & \\
\hline (b) & $\operatorname{PSR}(\%)$ & $\operatorname{FDR}(\%)$ & $\mathrm{AE}$ & $\operatorname{PSR}(\%)$ & $\operatorname{FDR}(\%)$ & $\mathrm{AE}$ \\
\hline DI & $98.2(5.2)$ & $0.7(0.7)$ & $0.3(0.1)$ & $98.3(5.0)$ & $1.1(1.3)$ & $0.3(0.1)$ \\
\hline $\mathrm{CA}-(2 / 6)$ & $78.0(8.2)$ & $0.8(0.7)$ & $0.7(0.1)$ & $89.3(8.7)$ & $28.1(18.2)$ & $1.5(0.7)$ \\
\hline CA- $(3 / 6)$ & $79.2(7.3)$ & $0.7(0.7)$ & $0.3(0.1)$ & $88.7(7.1)$ & $12.2(15.4)$ & $0.6(0.5)$ \\
\hline SCCA & $56.3(10.3)$ & $2.3(1.2)$ & - & & & \\
\hline DI-LS & $83.3(5.8)$ & $4.8(3.9)$ & $0.3(0.1)$ & & & \\
\hline (c) & $\operatorname{PSR}(\%)$ & $\operatorname{FDR}(\%)$ & $\mathrm{AE}$ & $\operatorname{PSR}(\%)$ & $\operatorname{FDR}(\%)$ & $\mathrm{AE}$ \\
\hline DI & $99.7(2.3)$ & $0.1(0.1)$ & $0.1(0.1)$ & $99.8(1.7)$ & $0.4(0.8)$ & $0.1(0.1)$ \\
\hline $\mathrm{CA}-(2 / 6)$ & $82.3(6.2)$ & $0.7(1.1)$ & $0.5(0.1)$ & $84.5(4.9)$ & $3.0(3.3)$ & $0.5(0.1)$ \\
\hline $\mathrm{CA}-(3 / 6)$ & $82.3(4.0)$ & $0.4(0.7)$ & $0.2(0.1)$ & $82.8(2.9)$ & $1.7(1.6)$ & $0.2(0.1)$ \\
\hline SCCA & $80.0(14.2)$ & $6.6(0.7)$ & - & & & \\
\hline DI-LS & $83.5(2.9)$ & $1.3(1.4)$ & $0.2(0.1)$ & & & \\
\hline
\end{tabular}


Table 3: We consider the same scenario as Table 2, but now the predictors $X_{k i j}=I\left(X_{k i j}^{\prime}>0\right)(k=1,2 ; i=1, \ldots, n ; j=20, \ldots, 25)$.

\begin{tabular}{|c|c|c|c|c|c|c|}
\hline \multirow[b]{2}{*}{ (a) } & \multicolumn{3}{|c|}{$T=(\log p) / 3$} & \multicolumn{3}{|c|}{$T=(\log p) / 6$} \\
\hline & $\operatorname{PSR}(\%)$ & $\operatorname{FDR}(\%)$ & $\mathrm{AE}$ & $\operatorname{PSR}(\%)$ & $\operatorname{FDR}(\%)$ & $\mathrm{AE}$ \\
\hline DI & $93.0(7.4)$ & $1.8(1.6)$ & $0.3(0.2)$ & $98.0(5.9)$ & $2.5(2.4)$ & $0.3(0.2)$ \\
\hline $\mathrm{CA}-(2 / 6)$ & $82.0(5.7)$ & $5.0(3.4)$ & $0.8(0.2)$ & $84.7(5.6)$ & $18.2(13.9)$ & $0.9(0.3)$ \\
\hline $\mathrm{CA}-(3 / 6)$ & $81.2(5.7)$ & $2.4(1.5)$ & $0.4(0.1)$ & $83.0(3.3)$ & $6.1(7.5)$ & $0.3(0.2)$ \\
\hline SCCA & $51.8(19.2)$ & $4.2(4.9)$ & - & & & \\
\hline DI-LS & $84.3(6.2)$ & $6.2(5.5)$ & $0.3(0.1)$ & & & \\
\hline (b) & $\operatorname{PSR}(\%)$ & $\operatorname{FDR}(\%)$ & $\mathrm{AE}$ & $\operatorname{PSR}(\%)$ & $\operatorname{FDR}(\%)$ & $\mathrm{AE}$ \\
\hline DI & $87.8(13.9)$ & $0.9(1.3)$ & $0.4(0.3)$ & $94.5(6.9)$ & $1.9(2.0)$ & $0.4(0.2)$ \\
\hline CA- $(2 / 6)$ & $78.7(9.2)$ & $2.7(1.7)$ & $0.9(0.3)$ & $82.7(4.7)$ & $27.6(16.1)$ & $1.6(0.7)$ \\
\hline $\mathrm{CA}-(3 / 6)$ & $80.2(6.6)$ & $1.1(1.0)$ & $0.4(0.1)$ & $83.0(5.8)$ & $11.2(15.7)$ & $0.6(0.6)$ \\
\hline SCCA & $59.5(13.2)$ & $3.0(2.1)$ & - & & & \\
\hline DI-LS & $83.0(5.8)$ & $4.2(3.4)$ & $0.4(0.1)$ & & & \\
\hline (c) & $\operatorname{PSR}(\%)$ & $\operatorname{FDR}(\%)$ & $\mathrm{AE}$ & $\operatorname{PSR}(\%)$ & $\operatorname{FDR}(\%)$ & $\mathrm{AE}$ \\
\hline DI & $97.5(6.9)$ & $0.2(0.2)$ & $0.2(0.2)$ & $98.8(4.3)$ & $0.4(0.5)$ & $0.2(0.1)$ \\
\hline CA- $(2 / 6)$ & $81.8(4.8)$ & $2.8(2.4)$ & $0.7(0.1)$ & $82.3(4.0)$ & $4.2(3.7)$ & $0.7(0.1)$ \\
\hline CA- $(3 / 6)$ & $82.0(4.5)$ & $0.9(1.5)$ & $0.2(0.1)$ & $83.0(2.3)$ & $1.5(1.4)$ & $0.2(0.1)$ \\
\hline SCCA & $86.0(12.9)$ & $6.8(0.7)$ & - & & & \\
\hline DI-LS & $83.5(1.7)$ & $1.3(1.5)$ & $0.3(0.1)$ & & & \\
\hline
\end{tabular}


Tables 2 and 3 show the simulation results for a scenario with normal errors and complete group structure. The nonzero parameters are $\alpha_{11}^{*}$, $\alpha_{16}^{*}, \alpha_{1(12)}^{*}, \alpha_{1(15)}^{*}, \alpha_{1(20)}^{*}$ and $\alpha_{21}^{*}, \alpha_{26}^{*}, \alpha_{2(12)}^{*}, \alpha_{2(15)}^{*}, \alpha_{2(20)}^{*}$. Let $\Phi(\cdot)$ be the distribution function of a standard normal variable. For $k=1,2$ and $i=1, \ldots, n$, the predictors in Table 2 are $X_{k i 3}=\Phi\left(X_{k i 3}^{\prime}\right)$ and $X_{k i j}=X_{k i j}^{\prime}$ $(j \neq 3)$, while those in Table 3 are $X_{k i 3}=\Phi\left(X_{k i 3}^{\prime}\right), X_{k i j}=I\left(X_{k i j}^{\prime}>0\right)$ $(j=20, \ldots, 25)$ and $X_{k i j}=X_{k i j}^{\prime}$ otherwise. The binary predictors in Table 3 violate the transnormal assumption in Fan et al. (2016). The responses are $Y_{k i}=X_{k i}^{\mathrm{T}} \alpha_{k}^{*}+0.7 \xi_{k i} X_{k i 3}$. Our DI method achieves the highest positive selection rates and the lowest false discovery rates. It also has the smallest absolute errors. Apparently, the CA-(3/6) and the DI-LS method are fairly likely to miss predictors that are relevant at some quantile levels: for the first approach only the conditional median is modeled and for the second one only the conditional mean. Our DI method, however, works well since it takes the heterogeneity into account and works with multiple quantile levels simultaneously. Another interesting observation in Table 3 is that $T=(\log p) / 3$ tends to underfit models compared to $T=(\log p) / 6$.

In Tables 4 and 5 we present the simulation results for the same scenario as in Table 2, but now the predictors have an incomplete group structure. The error variables in the two tables have a normal distribution (Table 4) and a $t$-distribution with three degrees of freedom (Table 5). The nonzero 
Table 4: We consider the same scenario as Table 2, but now the predictors have an incomplete group structure.

\begin{tabular}{|c|c|c|c|c|c|c|}
\hline & \multicolumn{3}{|c|}{$T=(\log p) / 3$} & \multicolumn{3}{|c|}{$T=(\log p) / 6$} \\
\hline (a) & $\operatorname{PSR}(\%)$ & $\operatorname{FDR}(\%)$ & $\mathrm{AE}$ & $\operatorname{PSR}(\%)$ & $\operatorname{FDR}(\%)$ & $\mathrm{AE}$ \\
\hline DI & $97.2(5.6)$ & $1.8(1.7)$ & $0.4(0.1)$ & $98.0(4.3)$ & $2.4(2.1)$ & $0.3(0.2)$ \\
\hline $\mathrm{CA}-(2 / 6)$ & $86.0(6.8)$ & $3.4(3.0)$ & $0.7(0.1)$ & $92.2(7.3)$ & $23.7(16.5)$ & $0.9(0.3)$ \\
\hline CA- $(3 / 6)$ & $84.6(5.4)$ & $2.2(1.9)$ & $0.4(0.1)$ & $87.2(4.8)$ & $7.6(8.6)$ & $0.4(0.2)$ \\
\hline SCCA & $45.0(12.5)$ & $5.6(4.4)$ & - & & & \\
\hline DI-LS & $88.6(5.3)$ & $7.1(5.4)$ & $0.4(0.1)$ & & & \\
\hline (b) & $\operatorname{PSR}(\%)$ & $\operatorname{FDR}(\%)$ & $\mathrm{AE}$ & $\operatorname{PSR}(\%)$ & $\operatorname{FDR}(\%)$ & $\mathrm{AE}$ \\
\hline DI & $91.3(9.7)$ & $0.8(0.9)$ & $0.4(0.1)$ & $96.6(6.5)$ & $2.0(2.0)$ & $0.4(0.1)$ \\
\hline CA- $(2 / 6)$ & $82.9(6.0)$ & $1.4(1.2)$ & $0.8(0.1)$ & $92.0(7.1)$ & $32.6(18.3)$ & $1.7(0.7)$ \\
\hline $\mathrm{CA}-(3 / 6)$ & $83.8(6.2)$ & $1.1(1.0)$ & $0.4(0.1)$ & $87.1(7.3)$ & $13.7(16.5)$ & $0.8(0.6)$ \\
\hline SCCA & $45.7(12.1)$ & $2.7(2.6)$ & - & & & \\
\hline DI-LS & $87.4(4.9)$ & $5.0(3.3)$ & $0.4(0.1)$ & & & \\
\hline (c) & $\operatorname{PSR}(\%)$ & $\operatorname{FDR}(\%)$ & $\mathrm{AE}$ & $\operatorname{PSR}(\%)$ & $\operatorname{FDR}(\%)$ & $\mathrm{AE}$ \\
\hline DI & $98.2(4.1)$ & $0.2(0.4)$ & $0.2(0.1)$ & $98.2(4.1)$ & $0.4(0.6)$ & $0.2(0.1)$ \\
\hline CA- $(2 / 6)$ & $85.2(5.3)$ & $1.1(1.5)$ & $0.6(0.1)$ & $87.1(5.2)$ & $3.6(4.1)$ & $0.6(0.1)$ \\
\hline $\mathrm{CA}-(3 / 6)$ & $85.8(5.0)$ & $0.9(1.1)$ & $0.3(0.1)$ & $87.7(3.5)$ & $2.4(1.9)$ & $0.2(0.1)$ \\
\hline SCCA & $67.1(10.2)$ & $6.3(0.8)$ & - & & & \\
\hline DI-LS & $87.9(3.6)$ & $1.2(1.4)$ & $0.3(0.1)$ & & & \\
\hline
\end{tabular}


Table 5: We consider the scenario from Table 4 with an incomplete group structure, but now the random errors follow a bivariate $t$-distribution with three degrees of freedom.

\begin{tabular}{|c|c|c|c|c|c|c|}
\hline \multirow[b]{2}{*}{ (a) } & \multicolumn{3}{|c|}{$T=(\log p) / 3$} & \multicolumn{3}{|c|}{$T=(\log p) / 6$} \\
\hline & $\operatorname{PSR}(\%)$ & $\operatorname{FDR}(\%)$ & $\mathrm{AE}$ & $\operatorname{PSR}(\%)$ & $\operatorname{FDR}(\%)$ & $\mathrm{AE}$ \\
\hline DI & $93.7(6.9)$ & $1.4(1.4)$ & $0.5(0.1)$ & $94.9(6.0)$ & $2.0(2.2)$ & $0.4(0.1)$ \\
\hline $\mathrm{CA}-(2 / 6)$ & $83.0(6.8)$ & $2.6(2.6)$ & $0.8(0.1)$ & $88.7(8.0)$ & $12.9(13.0)$ & $0.8(0.3)$ \\
\hline $\mathrm{CA}-(3 / 6)$ & $81.2(5.8)$ & $1.7(1.8)$ & $0.5(0.1)$ & $84.8(5.6)$ & $5.4(5.6)$ & $0.4(0.2)$ \\
\hline SCCA & $44.0(13.9)$ & $5.8(6.3)$ & - & & & \\
\hline DI-LS & $83.3(7.2)$ & $9.9(4.9)$ & $0.7(0.3)$ & & & \\
\hline (b) & $\operatorname{PSR}(\%)$ & $\operatorname{FDR}(\%)$ & $\mathrm{AE}$ & $\operatorname{PSR}(\%)$ & $\operatorname{FDR}(\%)$ & $\mathrm{AE}$ \\
\hline DI & $89.7(9.9)$ & $0.7(0.8)$ & $0.5(0.2)$ & $94.1(7.3)$ & $1.7(1.7)$ & $0.5(0.1)$ \\
\hline $\mathrm{CA}-(2 / 6)$ & $80.7(6.6)$ & $1.4(1.5)$ & $0.9(0.2)$ & $85.0(8.4)$ & $12.7(15.7)$ & $1.3(0.8)$ \\
\hline $\mathrm{CA}-(3 / 6)$ & $81.3(6.5)$ & $0.9(0.8)$ & $0.5(0.1)$ & $83.7(6.4)$ & $4.8(9.7)$ & $0.6(0.5)$ \\
\hline SCCA & $43.2(10.5)$ & $2.7(3.3)$ & - & & & \\
\hline DI-LS & $83.6(7.7)$ & $7.7(4.0)$ & $0.9(0.4)$ & & & \\
\hline (c) & $\operatorname{PSR}(\%)$ & $\operatorname{FDR}(\%)$ & $\mathrm{AE}$ & $\operatorname{PSR}(\%)$ & $\operatorname{FDR}(\%)$ & $\mathrm{AE}$ \\
\hline DI & $96.2(5.3)$ & $0.1(0.3)$ & $0.3(0.1)$ & $96.3(5.3)$ & $0.3(0.4)$ & $0.3(0.1)$ \\
\hline $\mathrm{CA}-(2 / 6)$ & $83.0(6.0)$ & $1.0(1.5)$ & $0.7(0.1)$ & $85.9(6.3)$ & $3.1(3.4)$ & $0.6(0.1)$ \\
\hline $\mathrm{CA}-(3 / 6)$ & $83.4(5.6)$ & $0.6(0.8)$ & $0.3(0.1)$ & $85.4(5.2)$ & $1.6(1.4)$ & $0.3(0.1)$ \\
\hline SCCA & $64.2(9.3)$ & $6.1(0.8)$ & - & & & \\
\hline DI-LS & $84.7(5.9)$ & $2.6(1.6)$ & $0.7(0.3)$ & & & \\
\hline
\end{tabular}


parameters are $\alpha_{14}^{*}, \alpha_{16}^{*}, \alpha_{19}^{*}, \alpha_{1(12)}^{*}, \alpha_{1(15)}^{*}, \alpha_{1(20)}^{*}$ and $\alpha_{21}^{*}, \alpha_{26}^{*}, \alpha_{2(12)}^{*}, \alpha_{2(15)}^{*}$, $\alpha_{2(20)}^{*}, \alpha_{2(25)}^{*}$. For $i=1, \ldots, n$ the predictors in the first experiment are $X_{1 i 1}=\Phi\left(X_{1 i 1}^{\prime}\right)$ and $X_{1 i j}=X_{1 i j}^{\prime}$ for $j \neq 1$. The predictors in the second experiment are $X_{2 i 3}=\Phi\left(X_{2 i 3}^{\prime}\right)$ and $X_{2 i j}=X_{2 i j}^{\prime}$ for $j \neq 3$. The responses are $Y_{1 i}=X_{1 i}^{\mathrm{T}} \alpha_{1}^{*}+0.7 \xi_{1 i} X_{1 i 1}$ and $Y_{2 i}=X_{2 i}^{\mathrm{T}} \alpha_{2}^{*}+0.7 \xi_{2 i} X_{2 i 3}$. Inspecting the quantities in the two tables, we see that our DI method again has higher positive selection rates and lower false discovery rates. Also it produces similar or smaller absolute errors than its competitors. We still observe that in both tables criterion $(4.7)$ using $T=(\log p) / 6$ selects larger models than that using $T=(\log p) / 3$ does. In Table 5 with $t$-distributed error variables, the absolute errors of the DI-LS approach based on least squares regression are significantly larger than those of our DI method, which corroborates the robustness of quantile regression when the distribution of the errors is heavy-tailed.

\section{Examples}

\subsection{Multiple experiments}

In this section we apply our method to data from a liver toxicity study (Bushel et al., 2007), which are available in the $\mathrm{R}$ package mixOmics (Rohart et al., 2017). In the study two groups of 32 male rats were exposed 
$5.1 \quad$ Multiple experiments

to non-toxic (50 or $150 \mathrm{mg} / \mathrm{kg}$ ) and toxic $(1,500$ or $2,000 \mathrm{mg} / \mathrm{kg}$ ) doses of acetaminophen (paracetamol), respectively. There is a data set for each group, which contains the rats' expression profiles of 3,116 genes and level of cholesterol. Due to the different experimental environments, the two data sets have different measurements. We want to identify the genes that significantly affect the response, namely the level of cholesterol on a logarithmic scale, based on aggregating the two data sets. To preprocess the data the genes are sorted by the absolute values of their correlation coefficients with the response in each set. The top 200 genes in each set are included in the analysis as covariates. We observe that the absolute values of their realizations are all below 2.05, which indicates that Assumption 1 is satisfied.

To fit sparse models, we minimize the objective function (2.4) using all data. We consider quantiles $\tau_{m}=m / 10$ for $m=1, \ldots, 9$ and use two different penalties, the SCAD penalty and the minimax concave penalty (MCP). The tuning parameters of the penalties are chosen using formula (4.7), i.e. as minimizers of the MQBIC, with $T=\log p / 6$. In addition, we take an approach based on random partitions: we divide each data set randomly into two parts, a training set of size 24 and a validation set of size 8. This is repeated 50 times. The training set is used to select parameters and obtain parameter estimates as before, i.e. by minimizing (2.4) with $\lambda$ 


\subsection{Multiple experiments}

chosen using (4.7). The prediction errors

$$
\sum_{k=1}^{K} \sum_{m=1}^{M} \sum_{i=1}^{n} \rho_{m}\left(Y_{k i}-X_{k i}^{\mathrm{T}} \widehat{\theta}_{k m}-\widehat{b}_{k m}\right)
$$

defined by the loss function are calculated based on the estimates from the training sets and data $X, Y$ from the validation sets. Here $\widehat{b}_{k m}$ is the estimated intercept in the conditional quantile $Q_{\tau_{m}}\left(X_{k}\right)$.

For comparison we also consider a combined analysis (CA), the SCCA method and the DI-LS method described in Section 4. The CA method in this section now considers 9 quantiles $\tau_{1}=1 / 10, \ldots, \tau_{9}=9 / 10$ instead of one single quantile as in Section 4. The data sets and the quantiles are treated separately, then the results are combined. The prediction errors for SCCA and DI-LS are calculated by

$$
\sum_{k=1}^{K} \sum_{i=1}^{n} M\left|Y_{k i}-X_{k i}^{\mathrm{T}} \widehat{\theta}_{k}^{\prime}-\widehat{b}_{k}^{\prime}\right| / 2 .
$$

Recall that the scale factor $M$ is the number of quantiles used by DI as well as CA. Here it is used to make the prediction errors comparable with those for DI and CA in (5.8), which sum up $K \times M \times n$ quantile loss functions. For the SCCA method $\widehat{\theta}_{k}^{\prime}$ and $\widehat{b}_{k}^{\prime}$ represent the slope vector and the intercept obtained from the unpenalized least absolute deviations regression of $Y_{k}$ on the selected subvector of $X_{k}(k=1, \ldots, K)$. For the DI-LS method the estimates are directly generated by the penalized least squares regression. We record the sizes of the models that are fitted using the entire data sets, 
5.1 Multiple experiments

Table 6: Analysis of the liver toxicity data. The sizes of the selected subset models (column 2) are based on all data, the average sizes and prediction errors (column 3 and 4) are based on the data using random partitions. The standard deviations are in parentheses. Here DI denotes the data integration method based on multiple quantiles, CA the combined analysis, SCCA the sparse canonical correlation analysis, DI-LS the data integration method based on the least squares regression, SCAD the smoothly clipped absolute deviation and MCP the minimax concave penalty.

\begin{tabular}{|lccc|}
\hline & All Data & \multicolumn{2}{c|}{ Random Partition } \\
& Model Size & Model Size & Prediction error \\
DI with SCAD & 2 & $3.30(1.54)$ & $1.19(1.06)$ \\
DI with MCP & 2 & $2.60(1.09)$ & $1.24(1.33)$ \\
CA with SCAD & 13 & $13.92(3.84)$ & $1.54(1.26)$ \\
CA with MCP & 11 & $14.86(3.88)$ & $1.40(0.98)$ \\
SCCA & 15 & $13.58(1.75)$ & $4.10(1.37)$ \\
DI-LS & 8 & $8.28(3.77)$ & $3.07(1.59)$ \\
\hline
\end{tabular}

and the simulated means and standard deviations of the model sizes and prediction errors otained from the 50 replications.

Table 6 shows the results of analyzing the liver toxicity data. When using the entire data sets, our DI method with both the SCAD penalty and the MCP penalty selects the same two covariates, which are also chosen by the combined analysis with either of the two penalties. Interestingly, the models fitted by SCCA or DI-LS do not include these two covariates. This difference suggests heterogeneity in the data, as both the SCCA and the DI-LS method tend to ignore covariates that affect responses only at certain 
quantile levels, but not globally. Using the random partition approach, our DI method generates models that are, on average, more sparse than those obtained from the competitors, with lower prediction errors.

\subsection{Multiple responses}

As a second application, now with a multivariate response vector, we analyze data sets of financial market indices from the $\mathrm{R}$ package FusionLearn (Gao et al., 2019). These data contain three correlated indices: the VIX index, the S\&P 500 index and the Dow Jones index. The VIX and the S\&P 500 are negatively correlated, while the S\&P 500 and the Dow Jones are positively correlated (Gao and Carroll, 2017). The covariates are 46 major international equity indices, North American bond indices and major commodity indices. In the analysis the transformation $\log \left(V_{t} / V_{y}\right) \times 100$ of each index is used, where $V_{t}$ and $V_{y}$ denote today's and yesterday's value. The training data set consists of 232 records of three years' market performances with three-day spacing between the values. As shown in Gao and Carroll (2017), the values are not autocorrelated at a 5\% significance level.

As before, we minimize the objective function (2.4) to select covariates and estimate parameters. The quantiles in (2.4) are $\tau_{m}=m / 20$ for $m=$ $1,2, \ldots, 19$. We again use the SCAD penalty and the MCP, and determine their tuning parameters with criterion (4.7). The SCAD penalty selects four 
Table 7: Analysis of the financial market indices. The figures are the prediction errors and the sizes of the selected submodels. The full model size is $p=46$. Here DI denotes the data integration method based on multiple quantiles, CA the combined analysis, UR is unpenalized regression, SCCA the sparse canonical correlation analysis, DI-LS the data integration method based on the least squares regression, SCAD denotes smoothly clipped absolute deviation and MCP minimax concave penalty.

\begin{tabular}{|lcccc|}
\hline & Model Size & \multicolumn{3}{c|}{ Prediction errors } \\
& & VIX & Dow Jones & S\&P 500 \\
DI with SCAD & 4 & 10052.8 & 523.4 & 307.2 \\
DI with MCP & 4 & 10020.6 & 522.4 & 309.2 \\
CA with SCAD & 20 & 10150.4 & 637.3 & 400.1 \\
CA with MCP & 20 & 10125.1 & 637.9 & 396.1 \\
UR & 46 & 13408.5 & 644.0 & 663.4 \\
SCCA & 6 & 12998.1 & 658.1 & 513.4 \\
DI-LS & 13 & 13996.3 & 720.5 & 499.5 \\
\hline
\end{tabular}


5.2 Multiple responses

covariates, which are the same as the four covariates selected by the MCP penalty. The competing methods are the combined analysis with the two penalties and unpenalized regression. The latter includes all 46 covariates in the model and generates estimators by minimizing the loss function (2.3) without a penalty term. We use the five fitted models for predictions based on a (different) validation data set with 464 records. Prediction errors for the three indices, that is $\sum_{m=1}^{M} \sum_{i=1}^{n} \rho_{m}\left(Y_{k i}-X_{k i}^{\mathrm{T}} \widehat{\theta}_{k m}-\widehat{b}_{k m}\right)$ for $k=1,2,3$, and model sizes are recorded in Table 7 . There we also list the results for SCCA and the DI-LS approach. The prediction errors for these two methods are $\sum_{i=1}^{n} M\left|Y_{k i}-X_{k i}^{\mathrm{T}} \widehat{\theta}_{k}^{\prime}-\widehat{b}_{k}^{\prime}\right| / 2$ with $\widehat{\theta}_{k}^{\prime}$ and $\widehat{b}_{k}^{\prime}(k=1,2,3)$ as in $(5.9)$.

Our DI method with both the SCAD penalty and the MCP outperforms the other five approaches, while DI with the SCAD penalty and DI with the MCP yield similar prediction errors. Apart from that, our DI method selects models that are considerably smaller than those from the competitors, i.e. it achieves more sparsity. As in Section 5.1, the two DI approaches and the two CA methods choose the same four predictors, while SCCA selects only one and DI-LS none of them. This again indicates heterogeity in the data, i.e. some predictors affect the responses only locally. The two empirical data examples in Sections 5.1 and 5.2 clearly demonstrate the advantages of our method, especially its ability to handle complex data. 


\section{Conclusion and discussion}

To the best of our knowledge this is the first time that a quantile regression approach has been applied to a data integration scenario with high dimensional data. By considering multiple quantiles simultaneously we obtain a global picture of the relationship between predictors and responses. A penalized estimator and an information criterion, which aggregate information from multiple experiments, were developed to select variables and to estimate model parameters. Our method copes with heterogeneity in the data. It successfully exploits the group structure in the parameter set across quantiles and experiments so that influential predictors can be identified.

In practice the quality and relevance of data may vary from one source to another. Therefore a weighted version of the loss function (2.3),

$$
\ell_{n}^{(w)}(\theta)=n^{-1} \sum_{k=1}^{K} w_{k} \sum_{m=1}^{M} \sum_{i=1}^{n} \rho_{m}\left(Y_{k i}-X_{k i}^{\mathrm{T}} \theta_{k m}\right)
$$

with weight vector $w=\left(w_{1}, \ldots, w_{K}\right)^{\mathrm{T}}$, may improve our estimator, which uses uniform weights. It would be worthwhile specifying and constructing such weights for data from different experiments.

The nonconvex penalty function associated with the $L_{1}$-norm has different properties compared to the penalty function associated with the $L_{2^{-}}$ norm employed by Gao and Carroll (2017), which forces parameters in the same group to be all zero or all nonzero. When the least squares approach 
is used, Jiang and Huang (2015) show that the penalty associated with the $L_{1}$-norm can be applied if the group structure is incomplete, i.e. both zero and nonzero parameters exist in the same group. This capacity is called a "bi-level selection" property. In this article we focus on groups of parameters to identify predictors that have an impact on one or more responses at some quantile levels. In the simulations of Section 4 we saw that the SCAD penalty with the $L_{1}$-norm actually performs well at the group level even if the group structure is incomplete. Theoretical properties of the $L_{1}$-norm in the quantile regression setting still need to be investigated in greater detail.

\section{Supplementary material}

- The proofs of the theoretical results and additional simulation results are in the Supplementary Material.

- All the programs of Section 4 and 5 are available at https://github. com/guorongdai/data_integration.

- The data in Section 5.1 are from the R package FusionLearn, while the data in Section 5.2 are from the $\mathrm{R}$ package mixOmics.

\section{Acknowledgments}

The authors thank the referees and an Associate Editor for their helpful comments, which helped us improve the paper. Dai and Carroll's research was supported by a grant from the National Cancer Institute (U01CA057030). 


\section{REFERENCES}

\section{References}

Belloni, A. and V. Chernozhukov (2011). $\ell_{1}$-penalized quantile regression in high-dimensional sparse models. Annals of Statistics 39, 82-130.

Bushel, P. R., R. D. Wolfinger, and G. Gibson (2007). Simultaneous clustering of gene expression data with clinical chemistry and pathological evaluations reveals phenotypic prototypes. BMC Systems Biology 1, 15.

Fan, J. and R. Li (2001). Variable selection via nonconcave penalized likelihood and its oracle properties. Journal of the American Statistical Association 96, 1348-1360.

Fan, J., L. Xue, and H. Zou (2016). Multitask quantile regression under the transnormal model. Journal of the American Statistical Association 111, 1726-1735.

Gao, X. and R. J. Carroll (2017). Data integration with high dimensionality. Biometrika 104, $251-272$.

Gao, X., Y. Zhong, and R. J. Carroll (2019). FusionLearn: Fusion Learning. R package version 0.1.1, available at https ://CRAN.R-project.org/package=FusionLearn.

Gu, Y. and H. Zou (2020). Sparse composite quantile regression in ultrahigh dimensions with tuning parameter calibration. IEEE Transactions on Information Theory $\mathbf{6 6}, 7132-7154$.

Jiang, D. and J. Huang (2015). Concave 1-norm group selection. Biostatistics 16, 252-267.

Koenker, R. (2005). Quantile Regression. Cambridge, UK: Cambridge University Press.

Koenker, R. and G. Bassett (1978). Regression quantiles. Econometrica 46, 33-50.

Lee, E. R., H. Noh, and B. U. Park (2014). Model selection via Bayesian information criterion for 


\section{REFERENCES}

quantile regression models. Journal of the American Statistical Association 109, 216-229.

Peng, B. and L. Wang (2015). An iterative coordinate descent algorithm for high-dimensional nonconvex penalized quantile regression. Journal of Computational and Graphical Statistics 24, 676-694.

Rohart, F., B. Gautier, A. Singh, and K.-A. Le, Cao (2017). mixomics: An R package for 'omics feature selection and multiple data integration. PLoS computational biology 13(11), e1005752. available at http://www.mixOmics.org.

Sherwood, B. and L. Wang (2016). Partially linear additive quantile regression in ultra-high dimension. Annals of Statistics 44, 288-317.

Wang, L., Y. Wu, and R. Li (2012). Quantile regression for analyzing heterogeneity in ultra-high dimension. Journal of the American Statistical Association 107, 214-222.

Witten, D. M. and R. J. Tibshirani (2009). Extensions of sparse canonical correlation analysis with applications to genomic data. Statistical applications in genetics and molecular biology 8, 1-27.

Yu, K., Z. Lu, and J. Stander (2003). Quantile regression: applications and current research areas. Journal of the Royal Statistical Society: Series D (The Statistician) 52, 331-350.

Yuan, M. and Y. Lin (2006). Model selection and estimation in regression with grouped variables. Journal of the Royal Statistical Society, Series B 68, 49-67.

Zellner, A. (1962). An efficient method of estimating seemingly unrelated regressions and tests for aggregation bias. Journal of the American Statistical Association 57, 348-368. 


\section{REFERENCES}

Zou, H. (2006). The adaptive lasso and its oracle properties. Journal of the American Statistical Association 101, 1418-1429.

Zou, H. and M. Yuan (2008a). Composite quantile regression and the oracle model selection theory. Annals of Statistics 36, 1108-1126.

Zou, H. and M. Yuan (2008b). Regularized simultaneous model selection in multiple quantiles regression. Computational Statistics \& Data Analysis 52, 5296-5304.

Department of Statistics, Texas A\&M University

E-mail: rondai@stat.tamu.edu

Department of Statistics, Texas A\&M University

E-mail: uschi@stat.tamu.edu

Department of Statistics, Texas A\&M University

E-mail: carroll@stat.tamu.edu 